María Andrea Delfino

Universidad Nacional de Rosario

\title{
Desocupación, trabajo doméstico y desigualdad: una mirada desde el uso del tiempo en Rosario, Argentina
}

\begin{abstract}
Resumen: Las transformaciones en el mercado de trabajo y en los sistemas de bienestar conllevan una fuerte tensión entre los recursos disponibles y las necesidades existentes en los hogares. Esa inadecuación entre recursos y necesidades es cubierta - en parte - con un incremento del trabajo no remunerado realizado al interior de los hogares. En este sentido, este artículo tiene por objetivo mensurar el tiempo asignado por un grupo de desocupados asistidos por el Estado al trabajo doméstico, describir el tipo de actividades básicas que componen este trabajo y caracterizar el reparto diferencial de esas actividades entre hombres y mujeres. Para alcanzar los objetivos planteados se recurrió a la metodología de uso del tiempo a través de la técnica del diario autoadministrado.
\end{abstract}

Palabras cloves: desocupación; trabajo doméstico; uso del tiempo.

Copyright $\odot 2012$ by Revista Estudos Feministas.

\section{Introducción}

Con el advenimiento de la Modernidad, dos dinámicas se entrelazaron para erigir a una específica noción de trabajo en un lugar hegemónico. La generalización de las relaciones de producción capitalistas y el proceso de escisión y especialización de los espacios en público/laboral y privado/doméstico entronizaron la noción de trabajo inscrita en la economía de mercado, a la vez que colocaron a las restantes formas de trabajo en un lugar subordinado.

Esta transformación dio lugar a que los procesos de producción que se realizan en cada una de las esferas tomen la forma de procesos aparentemente paralelos, independientes y sin sentido de relación. En la medida en que cada una de las esferas realiza una función específica y se establece una clara frontera entre ambas, los dos procesos 
1 Cristina CARRASCO, 1995.

${ }^{2}$ Cristina BRUSCHINI, 2006

${ }^{3}$ CARRASCO, 1995.

${ }^{4}$ CARRASCO, 1995.

${ }^{5}$ Dina VAIOU, 1991/1992 y 1995. de trabajo llegan a ser completamente extraños unos a otros. ${ }^{1}$ Sin embargo, el trabajo remunerado y el trabajo no remunerado realizado al interior del hogar se encuentran altamente integrados, constituyendo dos dimensiones del trabajo social que están enteramente relacionadas, ${ }^{2}$ o mejor, constituyendo dos aspectos de un único proceso. ${ }^{3}$

Por un lado, la esfera industrial requiere para la producción de mercancías, además de otras mercancías, de fuerza de trabajo. Esa fuerza de trabajo, esencial para el funcionamiento del capitalismo, es reproducida al margen de las normas de este sistema: su reproducción y mantenimiento se realizan en la esfera doméstica. De forma inversa, la esfera doméstica depende, para reproducir a los individuos y reproducirse a sí misma, de la producción industrial. Esta relación se concreta en la variable distributiva. Como estos beneficios generalmente no llegan a cubrir los costos de la reproducción de la fuerza de trabajo, es necesaria la realización de un trabajo adicional que permite transformar en consumibles aquellos bienes no consumibles adquiridos en el mercado. De manera adicional a este sinnúmero de interacciones, la esfera pública reinvierte sobre la esfera doméstica en forma de servicios públicos gratuitos. ${ }^{4}$ En este sentido, y para Dina Vaiou, ${ }^{5}$ los servicios públicos actúan modificando el tipo de trabajo doméstico, y condicionando tanto el tiempo necesario para su realización, como las rutinas diarias de las mujeres, que son quienes, principalmente, las realizan.

Son justamente estas interacciones las que permiten plantear que las reestructuraciones en curso en los mercados de trabajo y en los sistemas de bienestar acarrean una creciente tensión entre los recursos distribuidos y disponibles en las familias y los niveles de vida sedimentados en las costumbres, gustos y convenciones sociales, conllevando un incremento del trabajo no remunerado realizado en el interior de los hogares.

En este sentido, este artículo tiene por objetivo mensurar el tiempo asignado por los desocupados al trabajo doméstico, describir el tipo de actividades básicas que componen este trabajo y caracterizar el reparto diferencial de esas actividades entre hombres y mujeres. Para alcanzar los objetivos planteados se recurrió a la metodología de uso del tiempo a través de la técnica del diario autoadministrado. Fueron encuestados un total de 119 beneficiarias y beneficiarios del Programa Jefes y Jefas de Hogar Desocupados (en adelante, PJJHD) en la ciudad de Rosario, Argentina, entre marzo y septiembre de 2006. 
${ }^{\circ}$ Lourdes BENERÍA, 1984.

${ }^{7}$ BENERÍA, 1984.

\section{Algunas aproximaciones conceptuales y metodológicas a la noción de trabajo doméstico}

El trabajo no remunerado realizado al interior del hogar constituye el núcleo del proceso de reproducción social. Para Lourdes Benería, ${ }^{6}$ debe entenderse por reproducción un proceso dinámico de cambio vinculado a la perpetuación de los sistemas sociales. La misma incluye tanto la reproducción social como la biológica, de modo que su significado rebasa el de la reproducción de los seres humanos. En este sentido, es posible distinguir tres aspectos que se corresponden a diferentes niveles de abstracción teórica:

a) la reproducción social: se refiere a la reproducción de las condiciones sociales que sostiene un sistema social. En este caso, la cuestión fundamental está relacionada con el tipo de estructuras que se deben reproducir para que pueda darse esa reproducción social en su conjunto. Este aspecto de la reproducción implica la transmisión del acceso y el control de recursos económicos de una generación a otra;

b) la reproducción biológica, o procreación, consiste esencialmente en la crianza de los hijos. Aunque es un componente básico de la reproducción de la fuerza de trabajo, se puede diferenciar claramente de ésta; y

c) la reproducción de la fuerza de trabajo: no sólo se refiere al mantenimiento cotidiano de los trabajadores presentes y futuros, sino también a la asignación de agentes a determinadas posiciones en el proceso productivo. Es decir que, mientras la reproducción biológica es estrictamente cosa del desarrollo físico de los seres humanos, la reproducción de la fuerza de trabajo tiene que ver con el proceso por el cual esos seres humanos se convierten en trabajadores.

La distinción entre estos tres niveles de reproducción facilita el análisis del modo en cómo la mujer, en general, y la división del trabajo, en particular, se ven afectadas por la reproducción. Según Benería, ${ }^{7}$ dado el estado actual de la tecnología, sólo la reproducción biológica está inevitablemente ligada a la función reproductiva específica de la mujer. Sin embargo, todas las sociedades han asignado a la mujer otros dos aspectos fundamentales de la reproducción de la fuerza de trabajo, a saber, el cuidado de los hijos y el complejo de actividades asociadas con el mantenimiento diario de las fuerzas de trabajo dentro de la unidad doméstica. La razón de esto se encontraría, siempre dentro de la perspectiva de la misma autora, en el papel reproductor de la mujer; función que ha constituido la base de la asociación de la mujer con el cuidado de los niños y con otras tareas relacionadas con el mantenimiento cotidiano de la fuerza de 
${ }^{8}$ María Angeles DURAN, 2005b. 9 Rosario AGUIRRE, 2005; DURÁN, 2005b; y VAIOU, 1991/1992.

${ }^{10}$ Fabiola CAMPILLO, 2000.

"Antonella PICCHIO, 2001. trabajo. En esta asociación se encontraría la raíz de la separación entre la esfera en que primariamente se concentra la mujer y aquella en la que lo hace el varón.

En relación a los elementos que definen el trabajo doméstico, es posible señalar que una de las primeras conceptualizaciones sobre el mismo puede encontrarse en el estudio pionero de Margareth Reid de la década del 30. En él se considera trabajo doméstico a aquellas actividades no remuneradas realizadas en el hogar llevadas a cabo por y para sus miembros, actividades que podrían ser reemplazadas por bienes del mercado o servicios pagados, si circunstancias tales como los ingresos, las condiciones del mercado y las inclinaciones personales permiten que el servicio fuese delegado en alguien fuera del grupo familiar. En este sentido, el trabajo doméstico no remunerado se define por el denominado criterio de la tercera persona, esto es, por tratarse de un tipo de trabajo que podría ser realizado remuneradamente por una persona externa al hogar.

Esta conceptualización presenta dos limitaciones. La primera de ellas es que se centra, fundamentalmente, en las transformaciones más frecuentes y elementales del entorno físico del hogar y sólo incorpora una definición restringida de cuidados, ciñéndose a la prestación concreta y activa de cuidados personales. ${ }^{8}$ En este sentido, una serie de autores ${ }^{9}$ señalan que este tipo de definición desconoce conceptos esenciales para entender el trabajo de los responsables del hogar, tales como los conceptos de "dirección", "gestión" y "disponibilidad", difícilmente traducibles en estimaciones de esfuerzo, intensidad o tiempo. Este tipo de actividades relacionadas con los dominios simbólicos están próximas a tareas de dirección y a condiciones de disponibilidad, y son por ello mucho más difíciles de observar por observadores externos y de conceptualizar y percibir por los propios sujetos que las realizan. La segunda limitación que plantea este enfoque es que considera económicamente productivo a aquello que se monetariza, independientemente del valor que pueda tener el servicio o bien generado para resolver necesidades. ${ }^{10}$

Desde la perspectiva de Antonella Picchio, ${ }^{11}$ el contenido del trabajo de reproducción social no remunerado es el cuidado del mantenimiento de los espacios y bienes domésticos, así como el cuidado de los cuerpos, la educación, la formación, el mantenimiento de relaciones sociales y el apoyo psicológico a los miembros de la familia. En lo que respecta a las actividades concretas desarrolladas y a su peso relativo, éste refleja los cambios históricos y culturales, mientras, en lo que se refiere a sus funciones de fondo, sigue manteniendo una posición central en el proceso 
de reproducción social de la población, que interacciona con el papel de los servicios públicos y la producción de los bienes y servicios de mercado necesarios para la subsistencia de la población. Este trabajo, necesario tanto para quien lo recibe como para quien lo asigna, forma parte de la organización profunda de las condiciones de vida, sedimentada en prácticas históricas de relación entre hombres y mujeres, clases y generaciones.

Para la autora, las grandes funciones del trabajo de reproducción social no remunerado diferenciadas a escala del sistema son las siguientes:

1) ampliación de la renta monetaria en forma de nivel de vida ampliado, que también incluye la transformación de bienes y servicios por medio del trabajo de reproducción social no remunerado. La ampliación tiene en cuenta los aspectos cuantitativos del trabajo de reproducción no remunerado y permite sumarlo a la renta monetaria para definir el nivel de vida en término de bienes y servicios bajo la forma en la que efectivamente se utilizan;

2) expansión del nivel de vida "ampliado" en forma de una condición de bienestar efectiva, que consiste en el disfrute de niveles específicos, convencionalmente adecuados, de educación, salud y vida social. Esta tiene en cuenta los aspectos cualitativos del trabajo de reproducción social y, en particular, la inversión de sentido inherente al trabajo de cuidado de las personas; $y$, finalmente

3) reducción cuantitativa y cualitativa de la población trabajadora a los trabajadores y trabajadoras efectivamente empleados; en este caso, el trabajo no remunerado desarrollado en el ámbito doméstico y familiar sirve de apoyo para la selección, realizada en el mercado de trabajo, de las personas y las capacidades personales efectivamente utilizadas en los procesos productivos, facilitando, material y psicológicamente, los procesos de adaptación a los mismos y/o absorbiendo las tensiones que generan.

La ausencia de un intercambio mercantil en el caso del trabajo doméstico o trabajo de reproducción social familiar ha determinado la invisibilidad de una contribución fundamental a la riqueza social, pero también ha permitido

12 BRUSCHINI, 2006

${ }^{13}$ DURÁN, 2005a ocultar una parte significativa de los costos de reproducción. En este sentido, Cristina Bruschini ${ }^{12}$ señala que es fundamental considerar el tiempo del trabajo doméstico como tiempo para la reproducción social, entendiéndolo como básico para resolver algunos problemas de la acumulación capitalista que no se ecuacionan en el sistema generalizado de intercambios realizados a través de la moneda.

Para María Ángeles Durán, ${ }^{13}$ el conjunto del trabajo no remunerado que se realiza en las familias en un momento dado es el resultado del ajuste entre demanda y oferta. La 


\footnotetext{
${ }^{14}$ Dentro de esta actividad se incluye la preparación de los alimentos y de la mesa, así como también la limpieza de la cocina y/o de los utensilios.
}

demanda de trabajo no remunerado parte de cinco categorías sociales principales: los niños, los enfermos, los ancianos, los sobreocupados en la producción para el mercado y los autoconsumidores. Las tres primeras categorías tienen en común que son en gran medida insolventes, es decir, no pueden pagar a precio de mercado el trabajo de asistencia que necesitan, por lo que alguien (el Estado a través de los servicios públicos, los familiares y amigos, u otros) ha de hacerlo por ellos. Parte de la población que vende su tiempo en el mercado sí puede comprar trabajo de atención; este trabajo es generalmente proveído por personas de los niveles sociales más bajos. En tanto, el autoconsumo de trabajo es la respuesta de la mayor parte de la población de rentas medias y bajas - especialmente de las mujeres -, porque su nivel de salarios, la cantidad de trabajo que venden al mercado y otros componentes de tipo cultural no le permiten adquirir trabajo para su propia atención; pero en cambio, está en condiciones de producirlo por sí misma. En tanto, la oferta de trabajo no remunerado es producida principalmente por mujeres.

Las actividades no remuneradas realizadas en el ámbito del hogar abarcan un amplio espectro de tareas. Sin embargo, a los efectos de convertirlas en categorías analíticamente operativas se las agrupó en un número limitado de subconjuntos:

a) trabajo doméstico (en sentido restringido): es aquel tipo de trabajo que supone la producción y transformación de mercancías, y el cuidado y el mantenimiento de los espacios realizado en el interior del hogar de forma no remunerada. Es un tipo de trabajo que está sometido a la orientación de los quehaceres; es decir, sometido al tiempo que exigen las cosas y hace que la jornada no esté sometida a un horario estricto. Desde el punto de vista operativo, uno de los criterios más difundidos para definir y delimitar este conjunto de actividades es el denominado "criterio de la tercera persona", por tratarse de un tipo de trabajo que podría ser realizado remuneradamente por una persona externa al hogar. Es decir, el trabajo doméstico puede ser definido como aquel tipo de trabajo que produce bienes y/ o servicios que son susceptibles de transformación en prácticas de trabajo mercantil. Incluye las tareas de alimentación, ${ }^{14}$ limpieza y orden del hogar, limpieza y orden de la ropa y el calzado, coser y tejer, reparación y mantenimiento de la vivienda, cuidado de plantas y mascotas, cuidado de la huerta y animales de granja y reparación y mantenimiento de vehículos;

b) trabajo específico de cuidados (no pagados): este conjunto de trabajos se definen como la acción de cuidar a un niño $\circ$ una persona adulta $\circ$ anciana dependiente 
${ }^{15}$ Siempre que fue posible, es decir, siempre que fue especificado por el beneficiario encuestado, el tiempo invertido en dar la comida a los niños fue contabilizado dentro de las tareas de cuidado. La adopción de este criterio responde a la necesidad de ampliar la mirada sobre el trabajo doméstico y familiar apartándose de las visione más materiales ligadas a la transformación, e incluir las numerosas actividades de gestión, disponibilidad, cuidados, etc.

${ }^{16} \mathrm{PICCHIO}, 2001$

17 Juliana MARTíNEZ FRANZONI, 2005.

${ }^{18}$ El PJJHD fue creado en 2002, en el marco de una crisis económica, social y política sin precedentes en la historia argentina, y estuvo vigente hasta aproximadamente mediados de 2006. Para esa época el gobierno de Néstor Kirchner comenzó a imprimir una serie de reformulaciones a las políticas de asistencia. El PJJHD aseguraba un ingreso mensual mínimo de 150 pesos (30 euros aproximadamente) a familias en condiciones de pobreza cuyo jefe/a de hogar se encontrase desocupado y tuviera al menos un hijo menor de 18 años o discapacitados de cualquier edad. El beneficiario debía retribuir este ingreso con la realización de una contraprestación laboral o educativa. Estas características permiten incluir al PJJHD dentro de los programas de transferencias monetarias condicionadas. integrante de la familia para el desarrollo y el bienestar de su vida cotidiana. Incluye todas las tareas relacionadas con los niños (desde la alimentación al estudio, pasando por el juego o la atención sanitaria) y las tareas de cuidados de enfermos o personas ancianas. Si bien implica un trabajo material, se trata de actividades que dependen de manera muy importante de las relaciones interpersonales que se establecen entre la persona que provee los servicios de cuidado y quien los recibe. Al igual que el "trabajo doméstico (en sentido restringido)", este tipo de actividades son susceptibles de ser delegadas por los miembros de la familia en personas ajenas a la misma y mercantilizadas. El mismo agrupa las siguientes actividades: cuidado de personas enfermas y/o adultos mayores dependientes y a niños, ${ }^{15}$ así como también los desplazamientos necesarios para las tareas de cuidado; $y$

c) adquisición de bienes y servicios y gestiones relacionadas con el hogar y la familia: este subgrupo no sólo contempla todas aquellas actividades cuya realización permite la obtención de bienes y servicios externos imprescindibles para el consumo privado del grupo doméstico, sino también todas aquellas gestiones relacionadas con la familia y el hogar. Estas actividades, también denominadas "articuladoras del bienestar", "obligatorias" y/o "servicio de apoyo", fueron pensadas como formas de articulación, de enlace entre los ámbitos domésticos y públicos, ${ }^{16}$ o como formas de vincular la producción del bienestar, que tiene lugar en las familias, con las prácticas de asignación de recursos mercantiles y públicos. ${ }^{17}$ Incluye las compras, pagos y todo tipo de trámites.

\section{Características de la población estudiada y metodología de investigación}

Dentro de esta investigación fue considerada como desocupada aquella persona cuya condición de tal fue reconocida por las instituciones especializadas de acción social. Se trata, entonces, de desocupados asistidos por el Estado nacional y más específicamente beneficiarios del mayor programa regulatorio del desempleo de la historia argentina: el Programa Jefas y Jefes de Hogar Desocupados. ${ }^{18}$

Es importante señalar que los beneficiarios del PJJHD conjugan una situación particular. A diferencia de un subsidio al desempleo tradicional, la percepción de ese beneficio no estaba limitada en el tiempo, y sus beneficiarios debían retribuir contraprestando con diferentes tareas entre 4 y 6 horas por el beneficio monetario percibido. Adicionalmente, el monto recibido tenía una escasa significación económica, y en algunas oportunidades era complementado con algún trabajo informal. 
${ }^{19}$ Una descripción general de la metodología de uso del tiempo sus potencialidades y limitaciones pueden encontrarse en AGUIRRE, 2005; Andrea DELFINO, 2009; DURÁN, 1997; y Daniela PEIXOTO RAMOS, 2009.

20 Para delimitar el porcentaje de encuestas correspondientes a cada uno de los sexos se utilizaron como referencia los datos surgidos de la Encuesta Permanente de Hogares (EPH) que realiza el Instituto Nacional de Estadísticas y Censos (INDEC) para el Aglomerado Gran Rosario durante el segundo semestre de 2005 (periodo inmediatamente anterior a la realización del trabajo de campo). Para ese momento, en el Aglomerado Gran Rosario, la población con planes de empleo estaba compuesta por un $92,4 \%$ de mujeres y un $7,8 \%$ de hombres. Tal como señalara Julio César Neffa (2007), desde el inicio de la implementación del PJJHD la cantidad y la proporción de mujeres fueron superiores a la de varones, y esta diferencia fue acentuándose a medida que pasó el tiempo. Este proceso llegó a ser tan relevante que comenzó a hablarse de una paulatina feminización del Programa a lo largo del tiempo en todo el país. Sin embargo, en este análisis no puede dejar de mencionarse una intencionalidad (en un principio latente y con posterioridad manifiesta) desde e Estado de mantener a las mujeres protegidas. Parte de esta población con pocas posibilidades de empleabilidad, pero vinculada a la atención de la calidad de vida de los niños/as, comienza a ser paulatinamente derivada al denominado Programa Familias.
Para alcanzar los objetivos planteados se recurrió a la metodología de uso del tiempo a través de la técnica del diario autoadministrado. ${ }^{19}$ Fueron encuestados 119 beneficiarios del Programa Jefes y Jefas de Hogar Desocupados en la ciudad de Rosario, Argentina, entre marzo y septiembre de 2006. De las 119 encuestas realizadas, 107 corresponden a mujeres, mientas que las 12 restantes corresponden a hombres, todos ellos beneficiarios del PJJHD. ${ }^{20}$

Por las características propias del Programa y/o por los "criterios de elegibilidad" de los perceptores, los beneficiarios encuestados comparten las siguientes características: 1) todos los encuestados son argentinos; 2) excepto una beneficiaria, todos tienen al menos un hijo menor de 18 años a cargo o discapacitados de cualquier edad. La excepción señalada es producto del hecho que en una segunda etapa el Programa se hizo extensivo a desocupados jóvenes y a mayores de 60 años que no contaban con prestación previsional; y 3) para la fecha en la cual fue desarrollado el trabajo de campo (marzo y septiembre de 2006), los beneficiarios llevaban entre tres y cuatro años de permanencia dentro del Programa.

En relación a la edad de los beneficiarios encuestados es posible señalar que hay diferencias marcadas entre las mujeres y los hombres. Las primeras se encuentran concentradas fundamentalmente entre los 25 y los 49 años. Dentro de este rango, la mayor presencia es de las beneficiarias con edad entre 30 y 34 años. En el caso de los hombres la situación es diferente, ya que predominan los hombres mayores a 45 años.

Respecto del nivel de escolaridad de los beneficiarios encuestados, tanto en el caso de los hombres como de las mujeres, priman los beneficiarios con primaria completa, seguidos por los de secundaria incompleta en el caso de las mujeres y de secundaria completa en el caso del los hombres. En relación al estado conyugal de las beneficiarias, y si consideramos de forma agregada a las casadas y unidas de hecho, este grupo constituiría el más representativo, seguidas por las solteras. El único grupo con escasa presencia es el de las viudas, situación que puede ser explicada claramente por la edad de las beneficiarias que son fundamentalmente jóvenes y adultas. Entre los hombres beneficiarios entrevistados también predominan los casados y unidos de hecho.

Las preguntas relacionadas con el número de hijos y de la cantidad de personas convivientes en el hogar presentaron altos niveles (alrededor del $50 \%$ ) de no respuesta o de errores, lo cual no permite elaborar tendencias claras. Entre los que respondieron priman los hogares con cinco miembros y los beneficiarios con 2 y 3 hijos. 
21 Dinah RODRÍGUEZ CHAURNET, 2004.

${ }^{22}$ AGUIRRE, 2005.

${ }^{23}$ Ramón RAMOS TORRE, 1990.

${ }^{24}$ RAMOS TORRE, 1990.

${ }^{25}$ Soledad MURILLO, 2000.
Analizar el tiempo asignado por los desocupados asistidos por el Estado al trabajo doméstico requiere una serie de consideraciones desde el punto de vista metodológico. En este sentido, es importante destacar que tanto el trabajo doméstico (en sentido restringido) como el trabajo de cuidados no pagos carecen de horarios definidos, y la cantidad de tiempo de trabajo destinada a dichas actividades estará determinada por las necesidades de los miembros de la unidad familiar. Además, la disponibilidad se extiende a fines de semana y días feriados. De ahí que ni uno ni otro estén sujetos a horario fijo y requieran la disponibilidad de él o los agentes que los realizan todo el tiempo. ${ }^{21}$

Adicionalmente, es muy habitual que las tareas del hogar se realicen en forma simultánea, densificando el ritmo de trabajo. Desde la perspectiva de Rosario Aguirre, ${ }^{22}$ una de las mayores dificultades encontradas en la medición del trabajo no remunerado tiene que ver con la variabilidad de la ejecución de tareas y, sobre todo, en la acumulación de tareas simultáneas. Reforzando esta complejidad, diferentes investigaciones han señalado la fuerte tendencia por parte de los encuestados a no registrar la simultaneidad de tareas en el trabajo doméstico y familiar.

Finalmente, y muy relacionado con este último punto, es importante considerar que el trabajo de cuidados es un tipo de actividad que depende en gran manera de las relaciones interpersonales. Esta característica intrínseca hace que el mismo no sea fácilmente visualizado y/o reconocido, incluso por quienes lo realizan. De esto se deriva la tendencia por parte de los encuestados o informantes a subestimar su participación y el tiempo medio empleado. Dos razones podrían ser encontradas en la base de esta dificultad. La primera de ellas se encontraría en que es una actividad difusa y típicamente secundaria. ${ }^{23}$ Es difusa porque se puede presentar en múltiples variantes, situadas en ese amplio espectro que va desde el trabajo doméstico-familiar propiamente dicho al ocio. Esto determina que en muchas situaciones el cuidado de la prole no se considere una actividad laboral propiamente dicha. Asimismo, y siempre desde la perspectiva de Ramón Ramos Torre, ${ }^{24}$ el trabajo de cuidados es una actividad típicamente secundaria porque no tiende a realizarse de forma segregada, sino simultáneamente con otras y además subordinándola a ellas. Esto hace que no se perciba como tal y que quede desplazada por la relevancia de la actividad principal.

La segunda razón, de orden más general, puede ser encontrada en lo que Soledad Murillo ${ }^{25}$ denominó "domesticidad". Para la autora, esta concepción que supone la plena disposición para el otro se ha visto "encerrada en 
${ }^{26}$ AGUIRRE, 2005.

27 RAMOS TORRE, 1990

${ }^{28}$ DURÁN, 2005b. una urna de nobles celofanes" que ocultan una serie de servicios traducibles en términos productivos, pero intencionalmente diluidos en la excelsa valoración de lo femenino unido al desprendimiento de si, a olvido de si, en un ritual más cercano al don, al regalo, conforme a la regla social que otorga al significado "hogar" una calidez desprovista de trabajo y obligaciones.

Derivado de estas consideraciones metodológicas debemos reconocer que es factible medir con mayor o menor precisión el tiempo dedicado a las tareas domésticas típicas, pero que esta posibilidad de captación está, generalmente, ceñida a las transformaciones más frecuentes del entorno físico del hogar y a la prestación concreta y activa de los cuidados. Sin embargo, y como ya fue señalado, resulta difícil de captar la llamada "carga mental" ${ }^{26}$ que conlleva la gestión, disponibilidad, dirección y armonización de estas actividades en el tiempo y en el espacio. Este tipo de actividades relacionadas con los dominios simbólicos están próximas a tareas de dirección y a condiciones de disponibilidad, y son por ello mucho más difíciles de captar por observadores externos y de conceptuar y percibir por los propios sujetos que las realizan.

Para el análisis de los datos fue utilizado un repertorio limitado y simple de índices, los mismos constituyen los más usuales en este tipo de investigaciones: ${ }^{27}$

1. duración media genérica: cantidad media de tiempo que emplea en una determinada actividad el conjunto (o un subconjunto específico) de la población estudiada:

2. tasa de participación: porcentaje de individuos que participan realmente en una determinada actividad; y

3. duración media específica: cantidad media de tiempo que emplea en una determinada actividad el conjunto de individuos que participan realmente en ella.

La distinción crucial es, lógicamente, la que separa la duración media genérica de la duración media específica. La primera toma en consideración la media de tiempo haciendo abstracción de en qué medida la población estudiada participa realmente de la actividad, mientras que la segunda sólo toma en consideración a los participantes reales. Para el análisis, fueron utilizados índices sintéticos; es decir, aquellos que toman en consideración el tiempo real destinado a varias tareas superpuestas. De este tipo de índices surgen valores menores que los obtenidos por agregación. Por el contrario, los índices obtenidos por agregación plantean algunas dificultades ya que hay tareas que se realizan simultáneamente. ${ }^{28}$ Para el establecimiento de los diferentes índices fue contabilizado sólo el tiempo demandado por cada una de las actividades 
${ }^{29}$ Nicky R. POUW, 1998.

${ }^{30} \mathrm{PICCHIO}, 2001$.

${ }_{31} \mathrm{PICCHIO}, 2001$. consignadas por los beneficiarios encuestados como actividad principal. Es decir, en esta ocasión no se hará referencia a las pocas simultaneidades registradas por los beneficiarios encuestados.

\section{Tiempo, trabajo doméstico y desocupación}

En relación al trabajo no remunerado realizado al interior del hogar, surgen de los diarios de actividades dos elementos fundamentales. El primero de ellos - de larga constatación en estudios que privilegian los enfoques de género - se relaciona con la distribución de las tareas y del tiempo asignado, diferenciales por sexo en el trabajo doméstico en general. De los datos surgidos de los diarios de actividades es posible observar una amplia desigualdad existente en los tres subconjuntos de actividades tanto en lo que respecta a las tasas de participación como a las medias genéricas de tiempo. Si bien la mayor participación femenina es innegable en el trabajo doméstico (en sentido restringido) y en el trabajo de cuidados, ésta se acentúa aún más en las actividades relacionadas con la adquisición de bienes y servicios y gestiones relacionadas con el hogar y la familia.

En este sentido y de forma clara, los comportamientos económicos de las familias exceden las orientaciones del mercado ${ }^{29}$ y se presentan como el resultado de un proceso social complejo que requiere bienes y trabajos, y que está regulado por normas sociales que se han sedimentado a lo largo del tiempo y están connotadas por sus contextos locales. ${ }^{30}$

El segundo elemento de análisis se desprende de la tensión creciente entre los recursos distribuidos y disponibles en la familia y los niveles de vida sedimentados en las costumbres, gustos y convenciones sociales, la cual hace pensar que las reestructuraciones en curso en el mercado de trabajo y en los sistemas de bienestar se están traduciendo en un incremento del trabajo no remunerado realizado por las mujeres en los núcleos familiares. La presión sobre el trabajo no remunerado es permanente, ya que a éste le corresponde cubrir el desfase entre las rentas disponibles y las normas sociales de consumo y, en particular, entre las condiciones laborales y las condiciones de vida. ${ }^{31}$

En adelante nos detendremos en un análisis más detallado de cada uno de los tres subconjuntos, haciendo especial referencia a los datos surgidos del trabajo de campo.

Trabajo doméstico (en sentido restringldo)

Al interior de este subconjunto es posible observar una amplia variedad de situaciones. Mientras algunas de las 


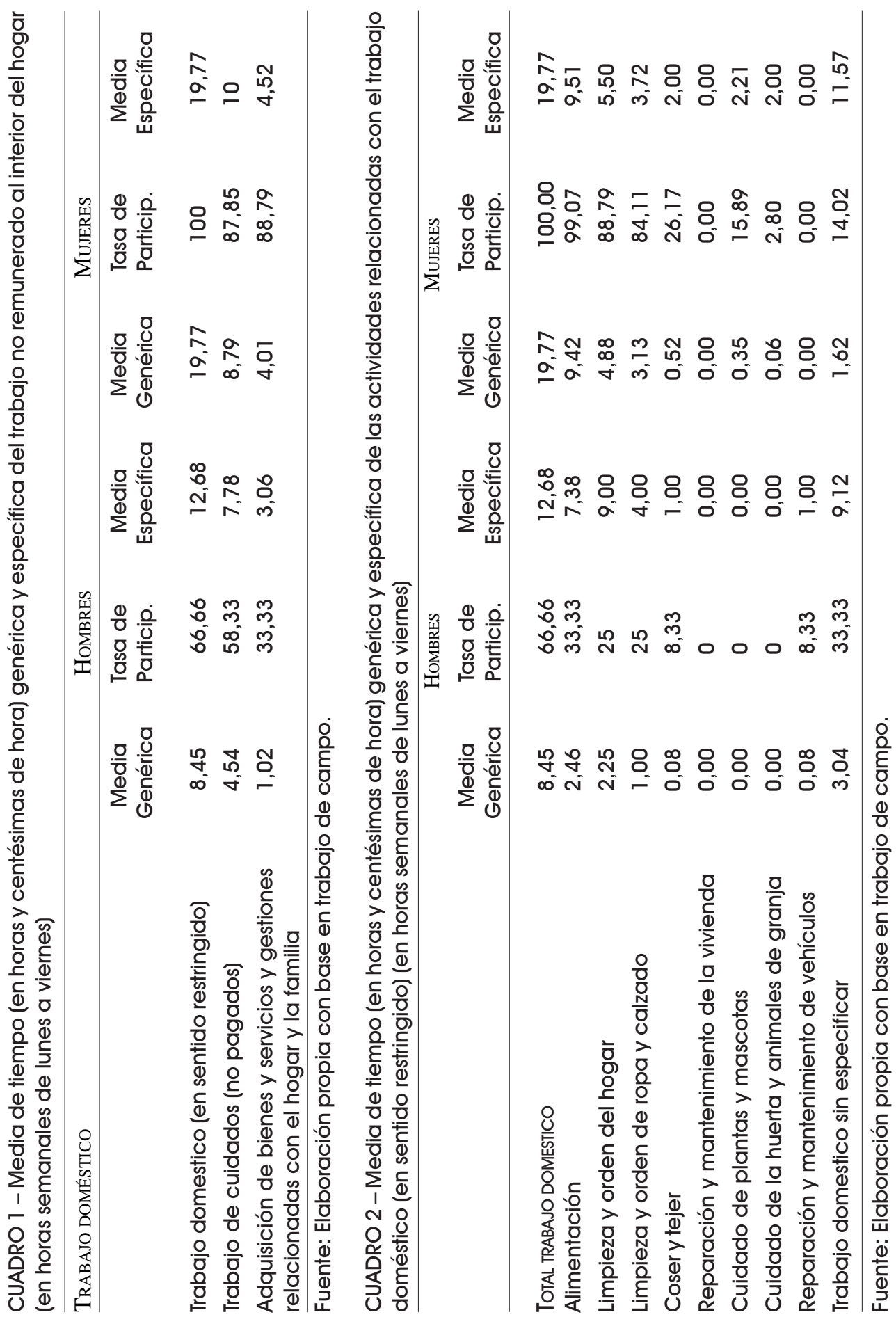

796 Estudos Feministas, Florianópolis, 20(3): 785-808, setembro-dezembro/2012 
actividades básicas que lo componen muestran elevadas tasas de participación y un número importante de horas asignadas, en otras la participación o dedicación a ellas es nula.

Las tareas relacionadas con la preparación de la comida, y en menor medida las relacionadas con la limpieza y orden del hogar y de la ropa, son las actividades que muestran las tasas más altas de participación tanto de las beneficiarias mujeres como de los beneficiarios hombres. Sin embargo y de forma conjunta, es posible observar, en estas mismas actividades, importantísimas desigualdades entre uno y otro sexo, ya que en los tres casos la participación de las mujeres supera ampliamente a la de los hombres. En resumidas cuentas, las tasas de participación masculinas más altas se encuentran en las tareas relacionadas con la preparación de la comida y, en menor medida, en las actividades relacionadas con la limpieza y orden del hogar y de la ropa, pero justamente se trata de actividades en las cuales las mujeres alcanzan tasas muy altas de participación, superando el $80 \%$.

Teniendo en cuenta el tiempo medio específico insumido por estas tres actividades, es interesante señalar que en dos de ellas (limpieza y orden del hogar, y limpieza y orden de ropa y calzado) el tiempo invertido por los hombres es mayor que el invertido por las mujeres.

En este sentido, los datos surgidos de los diarios de actividades muestran, en las tareas relacionadas con la preparación de alimentos y en aquellas relacionadas al orden y la limpieza (tanto del hogar como de la ropa), importantes desigualdades relativas a la participación diferencial de los sexos. De esta manera, se corrobora en todas esas actividades cotidianas centrales en la organización del hogar los resultados de una larga tradición de investigaciones. No acontece lo mismo si consideramos el tiempo medio específico asignado a estas actividades. En las dos actividades relacionadas con la limpieza y el orden (de ropa y del hogar), los hombres que se involucran en estas actividades lo hacen por más tiempo que las mujeres.

En tanto, aquellas actividades vinculadas con la autoproducción, como podrían ser las englobadas en coser y tejer y en cuidado de la huerta y animales de granja, son actividades que presentan una participación del $26,17 \%$ y el 2,80\%, respectivamente, de las beneficiarias mujeres, y una nula o escasísima participación de beneficiarios

${ }^{32}$ AGUIRRE, 2005 hombres. Según Aguirre, ${ }^{32}$ la expansión de la pobreza en las áreas urbanas actualiza el interés por tener presente estas actividades desarrolladas fundamentalmente por las mujeres como forma de contribuir al bienestar de sus familias. Para la autora, un interrogante a responder sería en qué medida 
${ }^{33}$ RAMOS TORRE, 1990.

${ }^{34}$ Catalina WAINERMAN, 2007.

${ }^{35}$ Karina BATTHYÁNY, 2009. algunas actividades domésticas han sido propulsadas fuera del hogar en los últimos años (mercantilizadas) y en qué medida han vuelto a ser "familiarizadas" como estrategia de bajar costos frente a la disminución de los ingresos de los hogares.

Una amplia literatura latinoamericana ha puesto en evidencia que los hogares adaptan sus estrategias de vida para hacer frente al desempleo y a la caída de los ingresos familiares a consecuencia de las políticas de ajuste aplicadas en la región. Así, el aumento de los precios de los bienes de consumo habría incentivado la autoproducción y orientado a los sectores de bajos ingresos hacia los mercados informales, más adaptados a su capacidad de compra. Los datos surgidos de los diarios de actividades, relacionados con las actividades de autoconsumo y la participación de los beneficiarios en trabajos remunerados informales relacionados con la venta o intercambio de productos ya elaborados o de elaboración doméstica, confluyen en confirmar para nuestro caso de estudio las tendencias más generales señaladas para América Latina.

Finalmente, las actividades ocasionales (tales como la reparación y el mantenimiento de vehículos y de la vivienda) presentan una participación nula de las mujeres pero también una participación muy escasa de los hombres. Las actividades relacionadas con el mantenimiento y la reparación de vehículos son las únicas actividades (tanto al interior del subgrupo como considerando al conjunto del trabajo no remunerado al interior del hogar) en las cuales la participación masculina supera a la femenina. Aún cuando es necesario relativizar esta participación por su escaso peso numérico, se trata de una tendencia corroborada por otras investigaciones. Tanto para Ramos Torre ${ }^{33}$ como para Catalina Wainerman ${ }^{34}$ son pocos los hogares en los que los varones no se ocupan de algo o mucho de las pequeñas reparaciones de plomería o electricidad que demanda el funcionamiento de la casa, del mantenimiento de vehículos y de otras tareas que no requieren ser hechas cotidianamente aunque sí precisan de ciertas "técnicas" o "calificaciones" supuestamente más extendidas entre los hombres que entre las mujeres.

\section{Trabajo específico de cuidados}

En este apartado analizaremos todas aquellas actividades de cuidado que son realizadas de manera "honoraria o benéfica" 35 por parientes en el marco de la familia. Dentro de este grupo son consignados también los desplazamientos derivados de esas tareas de cuidados.

En términos generales, las tareas específicas de cuidados no remunerados pueden estar dirigidas a adultos 
36 RAMOS TORRE, 1990.

37 Marie-Agnes BARRÈREMAURISSON, Sabine RIVIER y Olivier MARCHAND, 2001

${ }^{38}$ Barrère-Maurisson, Rivier y Marchand (2001) denominan a estas últimas "tiempo parental taxi".

\footnotetext{
39 RAMOS TORRE, 1990.
}

mayores, a enfermos y también a niños. Si bien es posible observar en los diarios de actividades de los beneficiarios tareas de cuidados destinadas a estos tres grupos de población, es innegable la supremacía que cobran las tareas de cuidados dirigidas a los niños en la cotidianeidad de los beneficiarios de planes sociales. Los denominados "criterios de elegibilidad de la población objetivo", es decir los criterios que definían quienes podían convertirse en beneficiarios del PJJHD, actúan reforzando la centralidad de estas actividades ya que uno de los requisitos era que el o la jefe/a de hogar desocupado/a tuviera al menos un hijo menor de 18 años o discapacitados de cualquier edad.

Desde la perspectiva de Ramos Torre ${ }^{36}$ el cuidado de la prole constituye una actividad social de primera importancia dada su trascendencia biológica y cultural. El "tiempo parental", como lo denominaron Marie-Agnes BarrèreMaurisson, Sabine Rivier y Olivier Marchand, ${ }^{37}$ agrupa todas las actividades efectuadas por los padres con o para sus hijos. Se trata, por una parte, del tiempo dedicado a ocuparse de los hijos: vestirlos y bañarlos, darles de comer, jugar con ellos, pasar con ellos tiempo fuera de casa, llevarlos a la escuela, acompañarlos en sus actividades extraescolares, ayudarlos con sus deberes escolares; por otra parte, se trata también del tiempo dedicado a los adolescentes: hablar, mirar juntos un programa de televisión, etc. En este sentido, sería posible diferenciar entre estas actividades a aquellas más vinculadas con la sociabilidad, con el trabajo doméstico en sentido restringido, con las actividades de seguimiento escolar y con los desplazamientos necesarios para estas tareas. ${ }^{38} \mathrm{Al}$ reagrupar esta amplia gama de actividades de características tan diferentes es posible observar que el trabajo específico de cuidados (sobre todo en el caso de los niños) aúna tanto componentes instrumentales como componentes expresivos si, como ocurre típicamente, los que lo realizan son el padre y la madre que encuentran una gratificación inmediata y no condicional en su práctica. ${ }^{39}$

A partir de los diarios de actividades puede observarse la permanencia de un patrón tradicional de comportamiento en relación a aquellas actividades vinculadas con el cuidado de los niños. Las tasas de participación de los beneficiarios en las actividades relativas al cuidado de los niños muestran una de las mayores distancias dentro de las denominadas actividades básicas. La media de tiempo específica destinada por las mujeres y por los hombres beneficiarios actúa confirmando la permanencia de este patrón. Mientras que las beneficiarias que declaran dedicar tiempo a tareas de cuidados de sus hijos lo hacen por más de 6 horas semanales (de lunes a viernes), en el caso de los hombres el tiempo dedicado cae a poco más de 4 horas. 


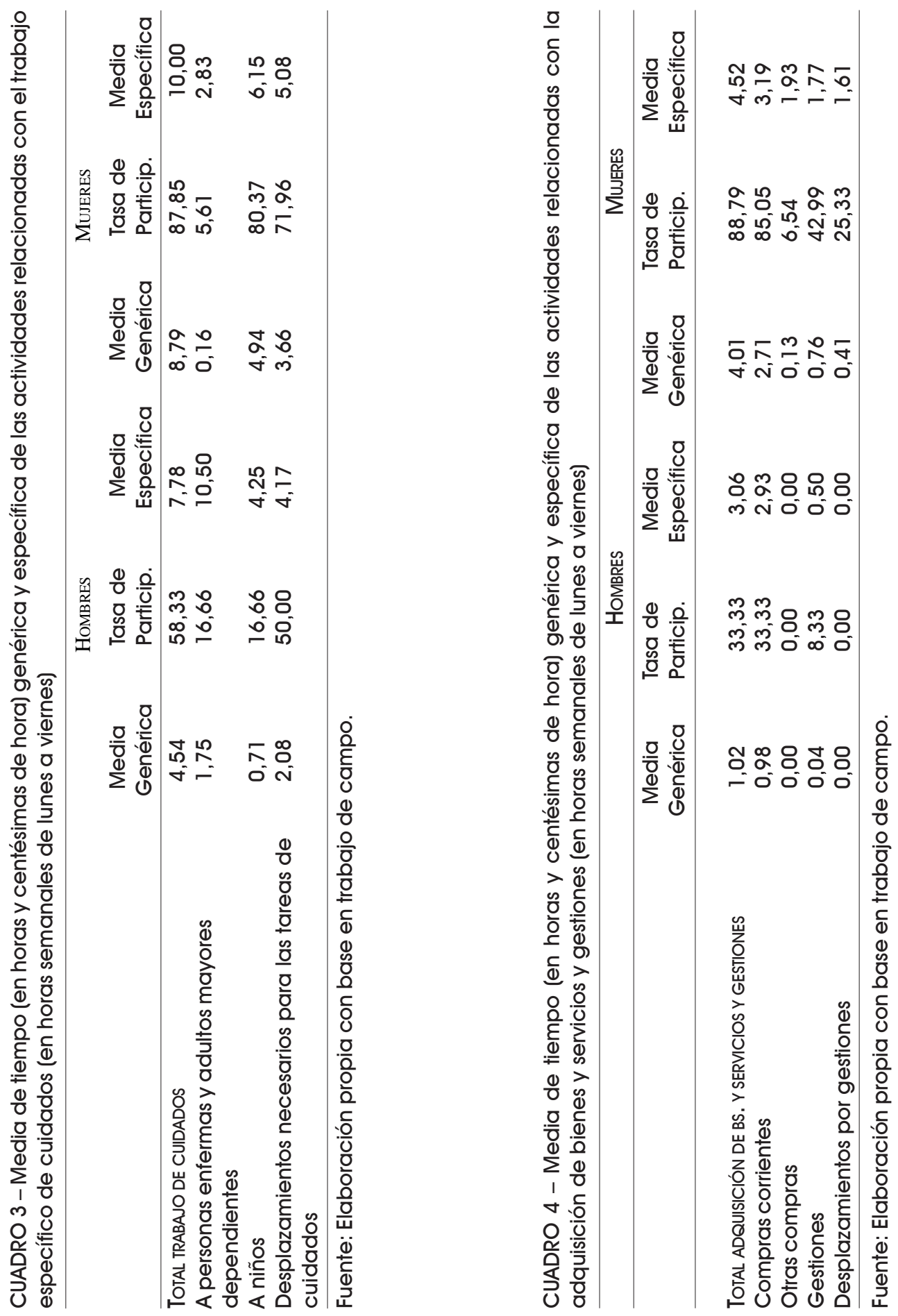

800 Estudos Feministas, Florianópolis, 20(3): 785-808, setembro-dezembro/2012 


\begin{abstract}
${ }^{40}$ Como señalamos anteriormente, la actividad básica que también mostraba una mayor participación masculina era de la "reparación y mantenimiento de vehículos" dentro del subgrupo "trabajo doméstico (en sentido restringido)". Sin embargo, en esta actividad la participación femenina era nula.
\end{abstract}

${ }^{41}$ DURÁN, 2005a.
Asimismo, es posible observar la recurrencia a inversiones de tiempo de otras mujeres (de la familia generalmente) como instancias de ayuda y colaboración en la crianza de los hijos. Es el caso de tres beneficiarias que manifiestan colaborar en el cuidado de sus nietos y de una beneficiaria que manifiesta (a través de las actividades registradas) desplazamientos constantes a la casa de su madre que la ayuda en el cuidado de su hija.

En el caso de los desplazamientos necesarios para las tareas de cuidados, la mayoría de ellos se vinculan con el cuidado de los niños y refieren a los traslados a la escuela, a las actividades de apoyo escolar (que los niños generalmente realizan en centros comunitarios del barrio), y a los comedores comunitarios, traslados para la búsqueda de turnos y/o controles médicos y traslados a actividades deportivas. En estas actividades las distancias entre las tasas de participación de las mujeres y de los hombres beneficiarios se acortan.

En relación al trabajo de cuidados a personas enfermas o adultos mayores, es la única actividad de la que, participando tanto hombres como mujeres, la mayor tasa de participación es la masculina. ${ }^{40}$ La media de tiempo específica de la actividad también es mayor para el caso de los hombres llegando a superar las 10 horas semanales (de lunes a viernes).

De los diarios de actividades de los beneficiarios del PJJHD se desprende que, en relación al trabajo específico de cuidados no remunerados, el lugar central está ocupado por los cuidados a los niños y adolescentes, y por los desplazamientos derivados de esas tareas de cuidados. Esta situación es clara en el caso de las mujeres beneficiarias. Los hombres beneficiarios, en cambio, muestran una participación equitativa en el cuidado de los niños y adolescentes, y en el cuidado de los enfermos y adultos dependientes; sin embargo, las asignaciones de tiempo son mayores en este último caso.

En este tipo de actividades es fundamental considerar que los contactos entre familiares que residen en distintos hogares no sólo son afectivos. En muchos casos conllevan fuertes transferencias de dinero y/o de trabajo no remunerado que hace posible la subsistencia de los hogares dependientes. Las transferencias las reciben sobre todo los hogares con personas ancianas, enfermas o con niños pequeños. Aunque no se moneticen, las transferencias de trabajo tienen una importancia mayor para el bienestar colectivo que las transferencias familiares de patrimonio y rentas. ${ }^{41}$ 


\footnotetext{
42 RAMOS TORRE, 1990, p. 128.

${ }^{43}$ La tasa de participación masculina en las compras corrientes constituye una de las más altas tasas de participación de los hombres considerando todas las actividades básicas incluidas en los tres subconjuntos. Sólo es igualada por la participación en las actividades relacionadas con la alimentación y superada por la participación en los desplazamientos relacionados con las tareas de cuidados.

${ }^{44}$ Entre las mujeres se evidencia una alta dispersión. Lo más frecuente $(19,8 \%$ de las beneficiarias) es una dedicación de 2,0 horas. Cuatro beneficiarias $(4,4 \%)$ son las que menos dedican $(0,5$ horas) y sólo una beneficiaria es la que dedica el máximo de tiempo a las compras corrientes $(9,5$ horas).

${ }^{45}$ RAMOS TORRE, 1990.
}

\section{Adquisición de bienes y servicios $y$ gestiones relaclonadas con el hogar y la familia}

Este conjunto de actividades es el que menor tiempo insume en horas semanales (de lunes a viernes) en relación con los otros dos subconjuntos de actividades (trabajo doméstico en sentido restringido y trabajo específico de cuidados). Esta característica se puede observar tanto para el caso de las mujeres como para el caso de los hombres. Tal como señaláramos, las mujeres beneficiarias dedican 19,77 horas semanales (de lunes a viernes) al trabajo doméstico en sentido restringido, 8,79 horas al trabajo de cuidados y sólo 4,01 horas a la adquisición de bienes y servicios y gestiones relacionadas con el hogar y la familia. El menor tiempo invertido en este tipo de actividades también puede ser observado en el caso de los hombres beneficiarios, quienes les dedican 8,45 horas, 4,45 horas y 1,02 hora, respectivamente.

En términos globales y agregados surgen otros dos elementos de análisis. El primero de ellos a ser señalado es que en las actividades vinculadas con la adquisición de bienes y servicios y gestiones relacionadas con el hogar y la familia se producen las menores tasas de participación masculinas. Sólo el $33,33 \%$ de los hombres beneficiarios encuestados han dedicado tiempo a este tipo de actividades durante las semanas de referencia. Si a este dato lo ponemos en relación con las tasas de participación femenina, surge un segundo elemento de análisis. La "crónica desigualdad que estructura el trabajo doméstico y familiar en su conjunto"42 aquí se ve reforzada. Las actividades vinculadas con la adquisición de bienes y servicios y gestiones relacionadas con el hogar y la familia son las que muestran una mayor distancia entre uno y otro sexo en lo relativo a sus tasas de participación.

Desagregando el subconjunto en sus actividades básicas, es posible señalar que son las compras corrientes las que alcanzan las mayores tasas de participación y, consecuentemente, ocupan una mayor cantidad de tiempo tanto para las mujeres como para los hombres ${ }^{43}$ beneficiarios del PJJHD encuestados, aun cuando se mantiene una amplia diferencia en la participación de unos y otros. Los hombres y las mujeres que participan de estas adquisiciones lo hacen con tiempos muy similares $(3,19$ horas en el período de lunes a viernes para las mujeres y 2,93 horas en el mismo período para los hombres beneficiarios). ${ }^{44}$

Es interesante observar que este tipo de comportamiento presenta algunos elementos comunes, pero también otros completamente opuestos al caso español analizado por Ramos Torre. ${ }^{45}$ Allí era posible observar el estrechamiento de las desigualdades entre varones y mujeres en sus 
${ }^{46}$ RAMOS TORRE, 1990.

${ }^{47}$ RAMOS TORRE, 1990. respectivas tasas de participación y sus medias de tiempo específico.

Para Ramos Torre, ${ }^{46}$ esto no se trataría del funcionamiento de un cierto principio de igualación de situaciones, sino del hecho que estas actividades tienen ciertas características que generan, con independencia de condicionamientos sexistas, este relativo estrechamiento de diferencias. Estas características distintivas están dadas por su periodicidad y rigidez temporal. Así, las compras - incluso las compras corrientes - no precisan realizarse con una periodicidad diaria, mientras que las actividades domésticas fundamentales sí lo requieren. Esto permite que las tasas de participación diaria de los sujetos directamente implicados en ellas se reduzcan de forma considerable. Consecuentemente, las mujeres, que son las que las realizan, alcanzan tasas de participación inferiores a las propias de trabajo doméstico, estrechando sus diferencias con los varones. Por otro lado, los condicionamientos espaciales a los que las actividades de compras están sometidas determinan un mínimo de tiempo muy rígido para la realización de la tarea. Ir de compras supone un desplazamiento fuera del hogar e invertir un mínimo innegociable de tiempo; contrariamente, en las actividades domésticas la fluctuación del tiempo para realizarlas es mucho mayor. Además, una vez invertido ese mínimo de tiempo imprescindible, las actividades de compra no precisan una alta inversión de tiempo diario. La conjunción de ambos factores define sus características de rigidez temporal: aquellos que las realizan se mueven en franjas de tiempo muy delimitadas. Esto explica que los varones y las mujeres inviertan, al realizarlas, cantidades muy similares de tiempo. Por esta razón, la igualación que aparece en este campo es resultado de su específica rigidez temporal.

El comportamiento de los beneficiarios del PJJHD en relación a la adquisición de bienes corrientes se aproxima al caso español en que las medias específicas de tiempo son similares para los hombres y las mujeres. La rigidez temporal característica de este tipo de actividades también puede ser aplicada para nuestro caso de análisis, constituyéndose así en el elemento central para explicar este dato. Sin embargo, no parece ocurrir lo mismo con la otra característica que Ramos Torre ${ }^{47}$ le adjudica a las compras corrientes: la periodicidad. Esta característica constituía en el caso español la lógica explicativa fundamental que permitía dar cuenta del estrechamiento de las desigualdades entre varones y mujeres en las tasas de participación en las actividades relacionadas con la adquisición de bienes.

Las compras corrientes de los beneficiarios encuestados, lejos de ser periódicas, se caracterizan por ser diarias, al menudeo y en diferentes comercios, incluso en muchos 
${ }^{48}$ Modalidad adoptada de las prestaciones alimentarias del Estado provincial a partir de 2005 en reemplazo de las tradicionales cajas alimentarias. El sistema de tickets o vales permitía a los beneficiarios canjearlos po comestibles en los comercios adheridos.

${ }^{49}$ Barry SCHWARTZ, 1992.

${ }^{50}$ David LEWIS y Andrew WEIGERT, 1992. casos se realizan dos veces al día. Sin lugar a dudas, los magros ingresos obtenidos a través de las asignaciones monetarias estatales y el tipo de trabajo remunerado realizado por los beneficiarios que conlleva ingresos irregulares no posibilitan la existencia de un día específico semanal destinado a las adquisiciones. La periodicidad diaria de las compras corrientes asimila estas actividades a aquellas otras, propias del trabajo doméstico (en sentido restringido) que también requieren de una periodicidad diaria.

En relación con las otras actividades básicas que conforman el subgrupo, es posible observar que los hombres prácticamente sólo participan de las compras corrientes, ya que muestran una participación casi insignificante en la realización de gestiones y una participación nula en las actividades relacionadas con la compras no corrientes les decir, con otras compras) y en los desplazamientos necesarios para la realización de las gestiones.

La insignificante participación masculina en las gestiones exteriores del grupo familiar llama en un principio la atención. Una serie de investigaciones habían señalado la participación igualitaria de hombres y mujeres o incluso la mayor participación masculina en este tipo de actividades. Esta diferencia puede ser comprendida por el tipo de gestiones mayoritariamente realizado por las y los beneficiarios del PJJHD, las cuales están fundamentalmente relacionadas con la procura de turnos médicos en los centros de salud públicos (actividad íntimamente ligada a las tareas de cuidado) y, en mucho menor medida, por pagos de impuesto y búsquedas de tickets o vales de alimentación. ${ }^{48}$ Es decir, en las tareas de gestión el peso mayoritario está constituido por gestiones relacionadas con los cuidados.

Si tenemos en cuenta que los desplazamientos que suponen las gestiones son contabilizados en una categoría aparte, es factible suponer que buena parte del tiempo invertido en estas actividades está relacionado con las esperas. Desde la óptica de Barry Schwartz, ${ }^{49}$ esperar es una señal reveladora de las relaciones de poder en los escenarios de interacción. La falta de coordinación de los horarios sociales lleva a que alguien deba esperar; quien espera es la persona que tiene relativamente poco poder y cuyo tiempo puede ser malgastado por los más poderosos. Para el autor, el análisis de las decisiones relativas a la espera es una manera útil de llegar a comprender el proceso mediante el cual se resuelven las incoherencias de los horarios sociales en la vida diaria. En una dirección similar, David Lewis y Andrew Weigert ${ }^{50}$ señalan que las esperas son una de las formas en que se resuelven los desajustes y los conflictos entre los diferentes tipos de tiempo social que operan en los distintos niveles de la estructura social. 


\section{Consideraciones finales}

El punto de partida para analizar el trabajo doméstico es considerar que los hogares adaptan sus estrategias de vida para hacer frente al desempleo y a la caída de los ingresos familiares como consecuencia de las políticas de ajuste aplicadas. Así, el aumento de los precios de los bienes de consumo genera cuatro tipo de prácticas: 1) "familiariza" (es decir, re-introduce en el hogar) actividades que habían sido propulsadas fuera del hogar, esto es, mercantilizadas; 2) incentiva la autoproducción; 3) orienta a los sectores de bajos ingresos hacia mercados informales, más adaptados a su capacidad de compra; y 4) propulsa hacia el ámbito comunitario actividades típicamente familiares como la comida.

Estos cuatro comportamientos impactan en el tiempo que necesita ser asignado para la realización de actividades dentro del hogar. Pero ese impacto es diferencial en términos de género. Las reestructuraciones en curso en el mercado de trabajo y en los sistemas de bienestar acarrean una tensión creciente entre los recursos distribuidos y disponibles en la familia y los niveles de vida sedimentados en las costumbres, gustos y convenciones sociales, conllevando un incremento del trabajo no remunerado que realizan las mujeres en los núcleos familiares. La presión sobre el trabajo no remunerado es permanente ya que a este le corresponde cubrir el desfase entre las rentas disponibles y las normas sociales de consumo y, en particular,

${ }^{51}$ PICCHIO, 2001.

52 RAMOS TORRE, 1990, p. 128. entre las condiciones laborales y las condiciones de vida. ${ }^{51}$

A partir de los datos surgidos de los diarios de actividades es posible observar una amplia desigualdad existente en los tres subconjuntos de actividades relativas al trabajo no remunerado realizado en el interior del hogar (trabajo doméstico en sentido restringido, trabajo de cuidados no pagados, y adquisición de bienes y servicios y gestiones relacionadas con el hogar y la familia) tanto en lo que respecta a las tasas de participación como a las medias genéricas y específicas de tiempo. Si bien la mayor participación femenina es innegable en el trabajo doméstico (en sentido restringido) y en el trabajo de cuidados, ésta se acentúa aún más en las actividades relacionadas con la adquisición de bienes y servicios y gestiones relacionadas con el hogar y la familia.

En este tercer subconjunto, la "crónica desigualdad que estructura el trabajo doméstico y familiar en su conjunto" 52 se ve reforzada, mostrando la mayor distancia entre uno y otro sexo en lo relativo a sus tasas de participación. Estos datos van a contrapelo de una serie de investigaciones que mostraban el estrechamiento de las 
desigualdades entre varones y mujeres en las tasas de participación y en las medias específicas de tiempo en las actividades vinculadas con la adquisición de bienes y servicios y gestiones relacionadas con el hogar y la familia.

Esta tendencia podría ser explicada, fundamentalmente, por las características que adquieren dos de las actividades básicas que conforman este subgrupo en la vida cotidiana de los beneficiarios del PJJHD. La primera de ellas, las compras corrientes, se caracteriza por ser diaria, al menudeo y en diferentes comercios e, incluso, en muchos casos se realizan dos veces al día. La segunda está relacionada con el tiempo de gestiones externas realizadas por las y los beneficiarios del PJJHD, las cuales están mayoritariamente direccionadas a la procura de turnos médicos en los centros de salud públicos (actividad íntimamente ligada a las tareas de cuidado) y en mucha menor medida por pagos de impuesto y búsquedas de tickets o vales de alimentación. Es decir, en las tareas de gestión, el peso mayoritario está constituido por gestiones relacionadas con los cuidados. Sin lugar a dudas, los magros ingresos obtenidos a través de las asignaciones monetarias estatales, el tipo de trabajo remunerado realizado que conlleva ingresos irregulares y la precariedad general en la que discurre la existencia de los beneficiarios son los elementos básicos en los que se asientan este tipo de prácticas.

\section{Referencias}

AGUIRRE, Rosario. "Trabajo no remunerado y uso del tiempo. Fundamentos conceptuales y avances empíricos. La encuesta Montevideo 2003". In: AGUIRRE, Rosario; GARCÍA SAINZ, Cristina; CARRASCO, Cristina. El tiempo, los tiempos, una vara de desigualdad. Santiago de Chile: Naciones Unidas, CEPAL, 2005. p. 9-31.

BARRÈRE-MAURISSON, Marie-Agnes; RIVER, Sabine; MARCHAND, Olivier. Tiempo de trabajo, tiempo parental. La carga parental: un trabajo de media jornada. Buenos Aires: CEIL-PIETTE, 2001.

BATTHAYÁNY, Karina. "Cuidado de personas dependientes y género". In: AGUIRRE, Rosario (Ed.). Las bases invisibles del bienestar social: el trabajo no remunerado en Uruguay. Montevideo: INE, INMUJERES, UDELAR, UNIFEM, 2009. p. 87123.

BENERÍA, Lourdes. Reproducción, producción y división sexual del trabajo. República Dominicana: Ediciones Populares Feministas, Ediciones CIPAF, 1984.

BRUSCHINI, Cristina. "Trabalho doméstico: inatividade econômica ou trabalho não-remunerado?". Revista Brasileira de Estudos de População, v. 23, n. 2, p. 331-353, jul./dez. 2006. 
CAMPILLO, Fabiola. "El trabajo no remunerado en la economía". Nómadas, n. 12, p. 98-115, abr. 2000.

CARRASCO, Cristina. "El treball doméstic y la reproducció social". Documents d'Analisi Geográfica, n. 28, p. 73 81,1995

DELFINO, Andrea. "La metodología de uso del tiempo: sus características, limitaciones y potencialidades". Espacio Abierto, Cuaderno Venezolano de Sociología, v. 18, n. 2, p. 119-218, abr./jun. 2009.

DURÁN, María Ángeles. "La investigación sobre uso del tiempo en España: algunas reflexiones metodológicas". Revista Internacional de Sociología, n. 18, p. 163-189, sept./dic. 1997.

"El trabajo no remunerado y las familias". In: CONSEJO NACIONAL DE LA MUJER; CONSEJO NACIONAL DE COORDINACIÓN DE POLÍTICAS SOCIALES. Decir mujer es decir trabajo: metodologías para la medición del uso del tiempo con perspectiva de género. Buenos Aires: Presidencia de la Nación, Consejo Nacional de Coordinación de Políticas Públicas, Consejo Nacional de la Mujer, Embajada de España, AECl, 2005a.

"Sociopsicología del trabajo no remunerado". In: CONSEJO NACIONAL DE LA MUJER; CONSEJO NACIONAL DE COORDINACIÓN DE POLÍTICAS SOCIALES. Decir mujer es decir trabajo: metodologías para la medición del uso del tiempo con perspectiva de género. Buenos Aires: Presidencia de la Nación, Consejo Nacional de Coordinación de Políticas Públicas, Consejo Nacional de la Mujer, Embajada de España, AECl, 2005b.

LEWIS, David; WEIGERT, Andrew. "Estructura y significado del tiempo social". In: RAMOS TORRE, Ramón (Comp.). Tiempo y sociedad. Madrid: Centro de Investigaciones Sociológicas (CIS) y Siglo XXI Ed., 1992. p. 89-13.

MARTÍNEZ FRANZONI, Juliana. "La pieza que faltaba: uso del tiempo y regímenes de bienestar en América Latina". Nueva Sociedad, n. 199, p. 35-52, sept./oct. 2005.

MURILLO, Soledad. "El tiempo de trabajo y el tiempo personal: un conflicto de intereses". In: FORMACIÓN Y ACREDITACIÓN EN CONSULTORÍA PARA LA IGUALDAD, BLOQUE TEMÁTICO, 8., Vitoria-Gasteiz: Emakunde, 2000.

NEFFA, Julio César, "Plan Jefes y Jefas de Hogar Desocupados y su evolución en Argentina (2002-2006)". In: V CONGRESO DE LA ASOCIACIÓN LATINOAMERICANA DE SOCIOLOGÍA DEL TRABAJO, Montevideo, Uruguay, del 18 al 20 de abril, 2007.

PEIXOTO RAMOS, Daniela. "Pesquisas de uso do tiempo: um instrumento para aferir as desigualdades de gênero". Revista Estudos Feministas, v. 17, n. 3, p. 861-870, set./ dez. 2009. 
PICCHIO, Antonella. 'Un enfoque macroeconómico 'ampliado' de las condiciones de vida". In: JORNADAS TIEMPOS, TRABAJO Y GÉNERO, Facultad de Ciencias Económicas de la Universidad de Barcelona, 2001.

POUW, Nicky R. La economía doméstica: desarrollo de una perspectiva alternativa. Madrid: WIDE, Coordinadora Estatal de ONGDS, 1998.

RAMOS TORRE, Ramón. Cronos dividido: uso del tiempo y desigualdad entre mujeres y hombres en España. Madrid: Ministerio de Asuntos Sociales, Instituto de la Mujer, 1990.

RODRÍGUEZ CHAURNET, Dinah. "Valoración económica y social del trabajo doméstico". In: CHÁVEZ CARAPIA, Julia del Carmen (Comp.). Perspectiva de Género. México: UNAM, 2004. p. 111-124.

SCHWARTZ, Barry. "Colas, prioridades y proceso social". In: RAMOS TORRE, Ramón (Comp.). Tiempo y sociedad. Madrid: Centro de Investigaciones Sociológicas (CIS) y Siglo XXI Ed., 1992. p. 307-324.

VAIOU, Dina. "Hogar y lugar de trabajo: la experiencia de las mujeres en el desarrollo urbano de Atenas". Documents d'Analisi Geografica, n. 19-20, p. 123-140, 1991/1992.

."El treball de les dones y la vida cuotidiana al sud d'Europa". Documents d'Analisi Geografica, n. 26, p. 219-231, 1995.

WAINERMAN, Catalina. "Familia, trabajo y relaciones de género". In: CARBONERO GAMUNDÍ, María Antonia; LEVÍN, Silvia (Comp.). Entre familia y trabajo, relaciones, conflictos y políticas de género en Europa y América Latina. Rosario: Homo Sapiens Ediciones, 2007. p. 147-175.

[Recebido em 14 de janeiro de 2011 reapresentado em 7 de novembro de 2011 e aceito para publicação em 8 de março de 2012]

\begin{abstract}
Unemployment, Housekeeping and Inequality: A Reading from a Time Use Survey in Rosario, Argentina

Abstract: The changes in the work market and in the welfare systems imply a strong tension between the available resources and the existing household needs. Such inadequacy between resources and needs is compensated -in part - by an increase in unpaid housework. In this sense, this article aims to estimate the time assigned by a group of unemployed people aided by the State to housekeeping; describe which kind of basic activities this work requires; and analyze the dissimilar division of activities between men and women. In order to attain the expected objectives, a time use survey was carried out through a self-administrated diary technique.

Key Words: Unemployment; Housekeeping; Time Use.
\end{abstract}

